\title{
HOXA11 plays critical roles in disease progression and response to cytarabine in AML
}

\author{
JEN-FEN FU ${ }^{1}$, LEE-YUNG SHIH ${ }^{2}$ and TZUNG-HAI YEN ${ }^{3}$ \\ ${ }^{1}$ Department of Medical Research, Chang Gung Memorial Hospital, Graduate Institute of Clinical Medical Sciences, \\ Chang Gung University, Taoyuan 333; ${ }^{2}$ Department of Internal Medicine, Division of Hematology-Oncology, \\ Chang Gung Memorial Hospital, Taipei $105 ;{ }^{3}$ Department of Nephrology, \\ Chang Gung Memorial Hospital, Chang Gung University, Taoyuan 333, Taiwan, R.O.C.
}

Received March 5, 2021; Accepted May 5, 2021

DOI: $10.3892 /$ or.2021.8101

\begin{abstract}
Lysine methyltransferase 2A (KMT2A, also known as $M L L)$ translocations $(M L L-t)$ are frequently associated with mutations in $R A S$ pathway genes in acute myeloid leukemia (AML). Previous findings with a mouse model showed that cooperation of $M L L / A F 10$ with tyrosine-protein phosphatase non-receptor type $11(\text { PTPN11 })^{G 503 A}$ accelerated leukemia development, but increased cytarabine (Ara-C) sensitivity of leukemia cells. To identify the genes responsible for reduced survival and Ara-C resistance, transcriptomic profiling between six pairs of mouse MLL/AF10(OM-LZ) leukemia cells harboring activating and wild-type $K R A S$ or PTPN11 was compared. A total of 23 differentially expressed genes (DEGs) with $>1.5$-fold-change between the paired cell lines were identified. The Gene Ontology (GO) terms overrepresented in these 23 DEGs included 'immune system process', 'actin filament binding', 'cellular response to interferon-alpha' and 'sequence-specific DNA'. Among the four genes (Hoxall, PR domain zinc finger protein 5, Iroquois-class homeodomain protein IRX-5 and homeobox protein PKNOX2) mapped to the GO term 'sequence-specific DNA', HOXA11 upregulation was associated with AML harboring $M L L$-t and $R A S$ signaling
\end{abstract}

Correspondence to: Dr Jen-Fen Fu, Department of Medical Research, Chang Gung Memorial Hospital, Graduate Institute of Clinical Medical Sciences, Chang Gung University, 5 Fu-Hsin Street, Kwei-San, Taoyuan 333, Taiwan, R.O.C.

E-mail: cgfujf@adm.cgmh.org.tw

Abbreviations: $M L L-\mathrm{t}, M L L$ translocations; AML, acute myeloid leukemia; Ara-C, cytarabine; DEGs, differentially expressed genes; GO, Gene Ontology; AL, acute leukemia; IL, interleukin; $\mathrm{MF}$, molecular function; BP, biological process; RT-qPCR, reverse transcription-quantitative polymerase chain reaction; cDNA, complementary DNA; i.p., intraperitoneally; BM, bone marrow; CCK-8, Cell Counting Kit-8; shRNA, short hairpin RNA

Key words: acute myeloid leukemia, $M L L$ translocation, activating tyrosine-protein phosphatase non-receptor type 11 mutation, Hoxa11, survival, cytarabine sensitivity mutations based on a meta-analysis using data deposited in Oncomine ${ }^{\mathrm{TM}}$ and analysis of the clinical samples in the present study. Microarray data revealed that only Hoxall was upregulated in those cells harboring activating PTPN11. Functional studies of Hoxall knockdown or overexpression in $M L L / A F 10(O M-L Z)$ cells revealed that Hoxall expression levels were associated with survival in vivo and Ara-C sensitivity/apoptosis in vitro. In addition, Hoxall regulated the expression of the apoptosis-related genes, $\mathrm{NF}-\kappa \mathrm{B}$ inhibitor $\alpha$, transcription factor p65 and transformation-related protein p53. Furthermore, the results of a meta-analysis using Heuser's AML dataset supported the finding that chemotherapy responders have higher expression levels of $H O X A 11$. These results indicated that the expression of $H O X A 11$ increased cell apoptosis and predicted an improved response to Ara-C in AML.

\section{Introduction}

Rearrangements of lysine methyltransferase 2A (KMT2A, also known as $M L L$ ) gene at chromosome 11q23 account for $\sim 10 \%$ of all acute leukemia (AL) cases, but are also present in most infant ALs and therapy-associated acute myeloid leukemia (AML), which were previously treated with topoisomerase II inhibitors for other cancers (1).

Although $>94$ fusion partner genes have been found to fuse with MLL, AF4 (AFF1), AF9 (MLLT3), ENL (MLLT1), AF10 (MLLT10), ELL and $A F 6$ (MLLT4) are the most frequent fusion partners found in Als (2). $M L L$ translocations ( $M L L-\mathrm{t})$ confer a poor prognosis in AL, especially $M L L / A F 6$ and $M L L / A F 10$ in AML $(3,4)$. $M L L$-t alters MLL methyltransferase activity and leads to dysregulation of $M L L$ downstream genes, such as $H O X A 7-A 10$, which subsequently impairs hematopoietic lineage commitment and induces leukemia development $(5,6)$. In addition to Hoxa7-Hoxal0 genes, sustained Hoxall expression has been detected in the $M L L / E N L$ immortalized myeloid cell line (7). Chromosomal translocation $\mathrm{t}(7 ; 11)$ (p15;p15) encoding NUP98/HOXA11 fusion has been recurrently detected in chronic myeloid leukemia and juvenile myelomonocytic leukemia $(8,9)$. HOXA11, HOXA10, HOXA7 and $H O X A 4$ are downregulated during monocyte-macrophage differentiation in a human leukemic THP-1 cell line (10). In 
contrast to HOXA7-HOXA10, the leukemogenic potential of HOXA11 is not well characterized. In addition to participating in leukemogenesis, the HOX family of genes are involved in organ development. In a homeobox swap experiment, it was discovered that Hoxalo could partially replace the role of Hoxall in regulating skeletal phenotypes and reproductive tract development (11). However, whether these different Hoxa genes are functionally interchangeable or complementary in leukemogenesis is not clear.

Cases of AL with $M L L$-t are frequently found to harbor $R A S$ pathway mutations, including $N-/ K-R A S$ and tyrosine-protein phosphatase non-receptor type 11 (PTPN11) activating mutations. The mutation rate of $K R A S$ ranges from 7.2 42.4\%, whereas that for NRAS is $5.3 \sim 24.7 \%$ and that for PTPN11 is $1 \sim 4.8 \%$ (12-15). The impact of $R A S$ pathway mutations on $M L L$-t AL is controversial. This is likely due to varied mutant allele frequencies of $R A S$ pathway mutations in patients $(16,17)$. We and others have established mouse models with results supporting that cooperation of $M L L$-t with activating $N-/ K-R A S$ or $P T P N 11$ mutations accelerate leukemia progression (18-22). Activating $N-/ K-R A S$ mutations constitutively activate the downstream signaling cascades controlling cell proliferation, apoptosis, differentiation and cell cycle progression (23). Activating PTPN11 mutations can induce myeloid cell hypersensitivity to growth factors, including granulocyte monocyte-colony stimulating factor and interleukin (IL)-3, and enhance cell cycle progression $(22,24,25)$. We also previously demonstrated that cooperation of $M L L-\mathrm{t}$ with activating PTPN11 mutations increased cytarabine (Ara-C) sensitivity in leukemia cells (22).

The underlying mechanism and key downstream players that accelerate leukemia development and Ara-C sensitivity by cooperating mutations have not been clearly illustrated. Thus, in the present study, transcriptomic profiles were compared between mouse MLL/AF10(OM-LZ) leukemia cells carrying wild-type and activating KRAS or PTPN11 to identify differentially expressed genes (DEGs) involved in survival and drug sensitivity. One such upregulated DEG, Hoxall, was further investigated to characterize its roles in leukemia cell differentiation, proliferation, survival and Ara-C sensitivity.

\section{Materials and methods}

Cell culture. The mouse $M L L / A F 10(O M-L Z)$ leukemia cell line (12G) and $M L L / A F 10(O M-L Z)$ cells harboring wild-type or activating $K R A S\left(K R A S^{G 12 C}\right)$ and wild-type or activating PTPN11 (PTPN11G503A) were generated by the retroviral transduction of genes to 5-fluorouracil (5-FU)-enriched C57BL/6J (B6) mouse bone marrow (BM) cells. The mice were purchased from the National Laboratory Animal Center. These different cell types were either generated in the current study (AKw1G) or in previous studies (AK2G, AK3G, APw1 and APm1) $(21,22,26)$ (Fig. 1A). All of these cell lines expressed the myelomonocytic markers. Mouse leukemia cells were cultured in the RPMI-1640 medium (Gibco; Thermo Fisher Scientific, Inc.) with 20\% fetal bovine serum (Hyclone; Cytiva), $2 \mathrm{mM}$ L-glutamine, $100 \mu \mathrm{M}$ 2-mercaptoethanol and $10 \mathrm{ng} / \mathrm{ml} \mathrm{IL-3} \mathrm{(R \& D} \mathrm{Systems,} \mathrm{Inc.)} \mathrm{for} \mathrm{maintenance} \mathrm{and}$ proliferation analysis.
Microarray analysis and Gene Ontology (GO) enrichment analysis. The total RNA of leukemia cells was prepared using TRIzol $^{\circledR}$ reagent (Gibco; Thermo Fisher Scientific, Inc.). The RNA was amplified, labeled and hybridized to the mouse genome 430A Array chip (12G vs. AK3G and $12 \mathrm{G}$ vs. AK2G), 4302.0 Array chip (12G vs. AK2G and APw-1 vs. APm-1), or Clariom D Array chip (APw-1 vs. APm-1 and AKw1G vs. AK3G) (Affymetrix; Thermo Fisher Scientific, Inc.) according to the manufacturer's instructions (this procedure was performed by staff of the Genomic Medicine Research Core Laboratory at Chang Gung Memorial Hospital, Linkou, Taiwan). Microarray data are available at the NCBI Gene Expression Omnibus (GEO) website (https://www.ncbi.nlm.nih.gov/geo/; accession nos. GSE82156 and GSE134586) or can be downloaded from Chang Gung University website (21). Differential Expression Analysis was performed using Transcriptome Analysis Console software version 4.0 (Affymetrix; Thermo Fisher). A heat map was obtained using Cluster version 3.0 (http://bonsai.hgc. jp/ mdehoon/software/cluster/) and Java TreeView version 1.1.6r4 (http://jtreeview.sourceforge.net). DEGs with $>1.5$-fold-change in paired MLL/AF10 leukemia cells harboring wild-type and oncogenic KRAS or PTPN11 were then functionally annotated with GO enrichment analysis using online Database for Annotation, Visualization and Integrated Discovery (DAVID) version 6.8 annotation tools (https://david.ncifcrf.gov/). Statistical significance was evaluated using Fisher's exact test and corrected by Bonferroni correction for multiple testing. The GO 'Molecular Function' (MF) or 'Biological Process' (BP) categories with $\mathrm{P}<0.05$ were considered statistically significant.

Validation of HOXA11 expression in patients with AML. To validate the differential expression and drug responsiveness of HOXA11 in MLL-t AML, a meta-analysis was performed using the Oncomine ${ }^{\mathrm{TM}}$ database (https://www.oncomine. org/), including Valk leukemia, Wouters leukemia, Balgobind leukemia, and Haferlach leukemia (27-30).

Reverse transcription-quantitative polymerase chain reaction ( $R T-q P C R)$. To evaluate the expression levels of target genes, the total RNA of mouse leukemia cells or AML patient BM cells was extracted using TRIzol ${ }^{\circledR}$ reagent (Invitrogen; Thermo Fisher). The total RNA was reverse transcribed into complementary DNA (cDNA) using random hexamers and SuperScript ${ }^{\mathrm{TM}}$ II reverse transcriptase (Thermo Fisher) according to the manufacturer's protocol. qPCR was performed using SYBR-Green PCR master mix (Applied Biosystems; Thermo Fisher Scientific, Inc.) and analyzed by ABI Prism 7900 system (Applied Biosystems; Thermo Fisher Scientific, Inc.). Primer sets were as follows: Mouse Hoxall, 5'-GAAAACCTCGCTTCCTCCGA-3' and 5'-ATAAGGGCA GCGCTTTTTGC-3'; mouse NF- $\mathrm{B}$ inhibitor $\alpha$ (Nfkbia), 5'-GAGACCTGGCCTTCCTCAAC-3' and 5'-TCTCGGAGC TCAGGATCACA-3'; mouse transcription factor p65 (Rela), 5'-TGGCTACTATGAGGCTGACCT-3' and 5'-TGGTCTGGA TTCGCTGGCTA-3'; mouse transformation-related protein p53 (Trp53), 5'-CCTCTCCCCCGCAAAAGAAA-3' and 5'-GGCCCTTCTTGGTCTTCAGG-3'; mouse Gapdh, 5'-TTC ACCACCATGGAGAAGGC-3' and 5'-GGCATGGACTGT GGTCATGA-3'; human HOXA11, 5'-CGTCTTCCGGCC ACACTGA-3' and 5'-AGACGCTGAAGAAGAACTCCC-3'; 
A
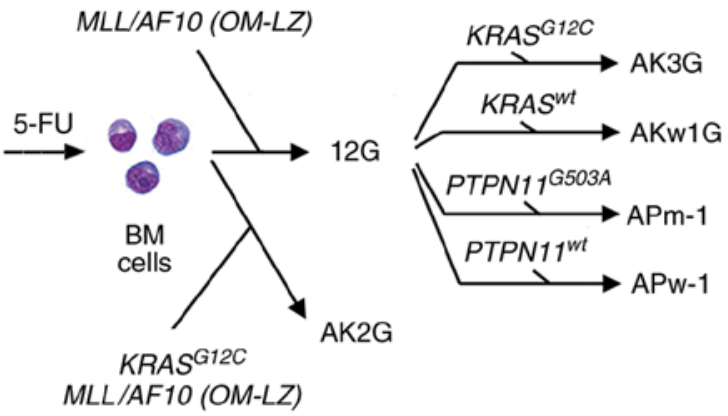

B
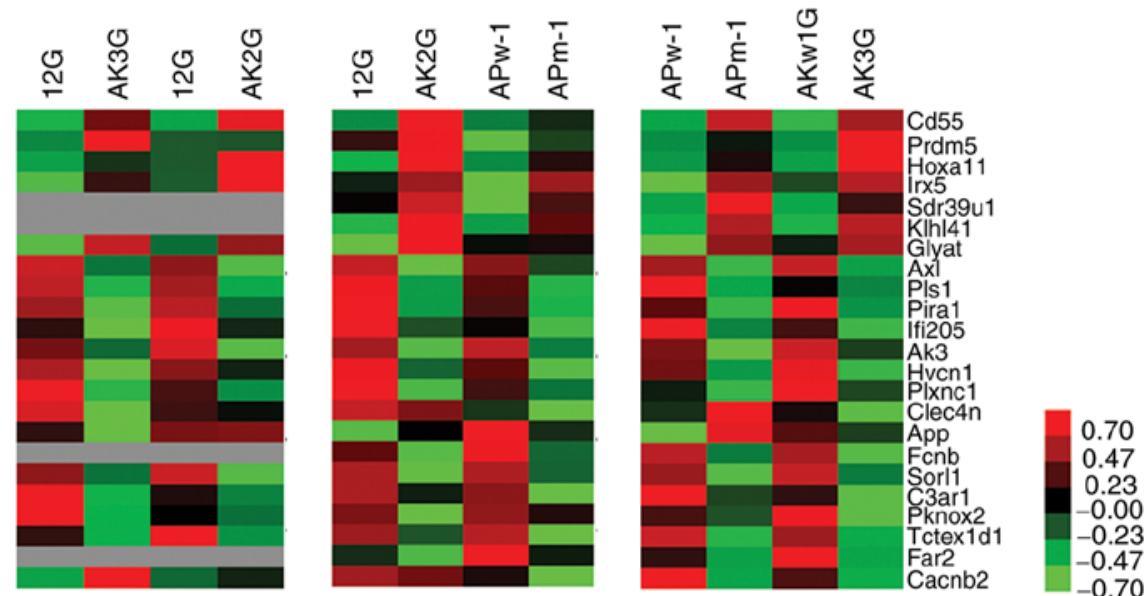

C

\begin{tabular}{|c|c|c|c|c|}
\hline Category & Term & $P$ value & Genes & Fold enrichmen \\
\hline BP & GO:0045087 innate immune response & 8.63E-04 & CD55, APP, FCNB, AXL, CLEC4N & 10.76 \\
\hline BP & GO:0002376 immune system process & 0.00822 & CD55, FCNB, AXL, CLEC4N & 8.99 \\
\hline MF & GO:0051015 actin filament binding & 0.00987 & PKNOX2, PLS1, CACNB2 & 18.88 \\
\hline BP & $\begin{array}{l}\text { GO:0035457 cellular response to } \\
\text { interferon-alpha }\end{array}$ & 0.01320 & AXL, IFI204 & 143.51 \\
\hline MF & GO:0043565 sequence-specific DNA & 0.03423 & PKNOX2, IRX5, HOXA11, PRDM5 & 5.25 \\
\hline $\mathrm{BP}$ & GO:0010468 regulation of gene expression & 0.04491 & APP, IRX5, HOXA11 & 8.39 \\
\hline
\end{tabular}

Figure 1. Identification of DEGs between paired MLL/AF10(OM-LZ) leukemia cells harboring wild-type and RAS pathway mutations. (A) Establishment of immortalized cell lines by retroviral transduction of 5-FU-enriched bone marrow cells with $M L L / A F 10(O M-L Z)$ alone (12G) or in combination with wild-type KRAS (AKw1G) or oncogenic KRASG12C (AK2G, AK3G), and wild-type PTPN11 (APw-1) or oncogenic PTPN11G503A (APm-1). (B) Heat map representing relative gene expression levels of 23 DEGs between paired cell lines based on cDNA microarray data. Different Affymetrix chips were used for the following paired cell lines: $12 \mathrm{G}$ vs. AK3G and $12 \mathrm{G}$ vs. AK2G (430A); $12 \mathrm{G}$ vs. AK2G and APw-1 vs. APm-1 (430_2), APw-1 vs. APm-1 and AKw1G vs. AK3G (Clariom D). Raw values were $\log 2$-transformed and centered relative to the median. A heat map was obtained using Cluster version 3.0 and Java TreeView version 1.1.6r4. The color bar depicts the color contrast level of the heat map, in which red and green indicates high and low expression, respectively. Grey indicates genes absent in the Affymetrix 430A chip. (C) Enriched GO terms of 'BP' and 'MF' for the 23 identified DEGs. All GO terms listed in the table show significant enrichment (all $\mathrm{P}<0.05$ ). Red and black indicate genes that are upregulated or downregulated, respectively, in $M L L / A F 10$ cell lines harboring $R A S$ pathway mutations. DEGs, differentially expressed genes; MLL, lysine methyltransferase 2A; GO, Gene Ontology; BP, biological process; MF, molecular function; PTPN11, tyrosine-protein phosphatase non-receptor type 11; BM, bone marrow; 12G, cells with $M L L / A F 10(O M-L Z)$ alone; AK3G, cells with $M L L / A F 10(O M-L Z)$ and oncogenic KRASG12C; AKw1G, cells with $M L L / A F 10(O M-L Z)$ and wild-type KRAS; APw-1, cells with $M L L / A F 10(O M-L Z)$ and wild-type PTPN11; APm-1, cells with MLL/AF1O(OM-LZ) and oncogenic PTPN11G503A; PTPN11, tyrosine-protein phosphatase non-receptor type 11.

and human $A B L, 5$ '-TGGAGATAACACTCTAAGCATAAC TAAAGG-3' and 5'-GATGTAGTTGCTTGGGACCCA-3'. The thermocycling conditions were as follows: $95^{\circ} \mathrm{C}$ for $2 \mathrm{~min}$; then 40 cycles of $95^{\circ} \mathrm{C}$ for $15 \mathrm{sec}$ and $65^{\circ} \mathrm{C}$ for $30 \mathrm{sec}$. The gene expression levels of mouse genes and human HOXA11 were normalized against the housekeeping genes Gapdh and $A B L$, respectively. Fold-change was calculated using the $2^{-\triangle \Delta C q}$ method (31). In the present study, leftover BM samples of clinical examination for initial diagnostic work-up of AML were used for gene expression analysis. Samples were obtained from patients admitted to Chang Gung Memorial Hospital (Taipei, Taiwan) between January 2002 and December 2010.
The inclusion criteria were as follows: Adults and children with AML with $M L L$-t. The exclusion criteria were as follows: Non- $M L L$-t AML specimens. Among the 114 cases with $M L L$ - $t$ AML (56 men and 58 women, age range $0-84$ years, median 27 years), it was determined that eight cases with $M L L / A F 10$ or $M L L / A F 9$ had sufficient remaining specimens available for HOXA11 expression level analysis. Of these eight cases, five had KRAS mutations and one had a PTPN11 mutation.

Western blot analysis. Total cell lysate from $5 \times 10^{6}$ leukemia cells was prepared by direct lysis of cells with RIPA buffer [20 mM Tris-Cl (pH 7.5) $150 \mathrm{mM} \mathrm{NaCl}, 1 \%$ Triton X-100, 
$1 \%$ Nonidet P-40, $0.5 \%$ sodium deoxycholate, $0.1 \%$ sodium dodecyl sulfate, $0.5 \mathrm{mM}$ PMSF]. The amount of total protein was assayed using a Pierce ${ }^{\circledR}$ BCA Protein Assay (Pierce; Thermo Fisher Scientific, Inc.). The lysates ( $20 \mu \mathrm{g} /$ lane) were electrophoresed on $10 \%$ polyacrylamide gel, and subsequently transferred to an Immobilon membrane (EMD Millipore). The membrane was blocked in $5 \%$ bovine serum albumin at $4^{\circ} \mathrm{C}$ (Sigma-Aldrich; Merck KGaA) for $1 \mathrm{~h}$, and then incubated with primary antibodies at $4^{\circ} \mathrm{C}$ overnight against mouse Hoxa11 (1:5,000; cat. no. NBP1-80228; Novus Biologicals, Ltd.), $\beta$-actin or Gapdh $(1: 10,000$; cat. nos. sc- 47778 or sc-32233; Santa Cruz Biotechnology, Inc.), followed by incubation for $2 \mathrm{~h}$ at room temperature with horseradish peroxidase-conjugated anti-mouse or anti-rabbit $\operatorname{IgG}$ secondary antibodies (1:5,000; cat. nos. C04001 or C04003; Croyez Bioscience Co., Ltd.). Western blots were developed with a Western Lightning Plus ECL kit (PerkinElmer, Inc.) and the images were visualized by Analytik Jena $^{\mathrm{TM}}$ UVP ChemStudio PLUS and VisionWorks ${ }^{\mathrm{TM}}$ software (version 9.0; Analytik Jena US LLC).

In vivo leukemogenesis. The in vivo leukemogenic potential of the leukemia cell lines was determined by BM transplantation assay using male B6 mice ( $\mathrm{n}=45$, age, $6-8$ weeks; weight, 20-24 g). Mice were maintained in pathogen-free devices under a controlled animal housing conditions (temperature $20 \pm 3^{\circ} \mathrm{C}$, humidity $60-70 \%$ with a 12 -h light /dark cycle and access to food and water ad libitum). Briefly, leukemia cells (APm1-shV, APm1-shH11-2, 12G-V, and 12G-H11) were intraperitoneally (i.p.) injected into mice $\left(1 \times 10^{6}\right.$ cells $/ 100 \mu 11 \mathrm{X}$ PBS/mouse; $\mathrm{n}=10$ mice for each leukemia cell line) that had received a sublethal dose of $\gamma$-irradiation (5.25 Gy) unless otherwise stated. Mice i.p. injected with normal saline $(\mathrm{n}=5)$ served as controls. To monitor leukemia development, peripheral blood (100 $\mu \mathrm{l})$ was collected weekly for cytologic analysis and complete blood count measurement using Hemavet 950 (Drew Scientific; Erba Diagnostics, Inc.) or BC-5000 (Mindray Medical International Limited) hemocytometers. Mice were sacrificed when moribund (7-15 weeks post-injection). The moribund state was defined as mice displaying leukocytosis, with hunched posture, weakness, shortness of breath and 20\% weight loss. Mice were euthanized by an i.p. injection of Zoletil $(50 \mathrm{mg} / \mathrm{kg}$ ) and Rompun (xylazine, $10 \mathrm{mg} / \mathrm{kg}$ ) or by inhalation of isoflurane (3-5\%), followed by cervical dislocation (32). BM, peripheral blood, ascites, organs and tumor masses were collected and weighed.

Gene knockdown by short hairpin RNA (shRNA). To generate stable gene knockdown cell lines, AK3G or APm-1 cells were infected with lentivirus expressing shRNA against Hoxall [The RNAi Consortium (cat. nos. TRCN0000413738 and TRCN0000417739)] at a multiplicity of infection of 1 and selected in RPMI-1640 medium containing puromycin $(2.5 \mu \mathrm{g} / \mathrm{ml})$ for a total of 2 weeks. A third generation system was used. Cells stably transfected with blank lentiviral vector pLKO_025 were used as negative controls. All lentiviruses were obtained from the National RNAi Core Facility at the Institute of Molecular Biology/Genomic Research Center, Academia Sinica (Taiwan).

Ectopic expression of Hoxall. The full-length Hoxall gene $(\sim 1 \mathrm{~Kb})$ was amplified from cDNA of AK3G cells by PCR using the following primers: 5'-GAAGATCTCCCAAGGTAG CCCAATGATG-3' and 5'-CCGCTCGAGCCAGTAGGC TGGAGCCTTAG-3'. The PCR product was digested with restriction enzymes $B g l \mathrm{II}$ and $\mathrm{XhoI}$, and was subsequently cloned into the $B g l \mathrm{II}$ and $\mathrm{XhoI}$ sites of the retroviral vector pMSCVpuro (Clontech Laboratories, Inc.). The fidelity of nucleotide sequences of the Hoxall gene was confirmed by Sanger sequencing. Plasmid DNAs of pMSCV-Hoxall and pMSCVpuro were transfected into EcoPack2-293 cells (Invitrogen; Thermo Fisher Scientific, Inc.) to package retroviruses. Viral titer was determined by infection of NIH/3T3 (American Type Culture Collection; ATCC ${ }^{\circledR}$ CRL-1658 ${ }^{\mathrm{TM}}$ ), a murine fibroblast cell line, with serial diluted supernatant to generate puromycin-resistant colonies. To generate $12 \mathrm{G}$ cell lines with ectopic expression of Hoxall (12G-H11-1 and 12G-H11-2), 12G cells were mixed with retroviruses at a 1:1 ratio and selected in RPMI complete medium containing puromycin $(2.5 \mu \mathrm{g} / \mathrm{ml})$ for a total of 2 weeks. The cells transduced with MSCVpuro retroviruses were used as negative controls (12G-V1 and 12G-V3). The presence of Hoxall gene was confirmed by PCR amplification of the $1-\mathrm{Kb}$ product from the genomic DNA of 12G-H11 cell lines using cloning primers. The fidelity of nucleotide sequences of Hoxall was confirmed by Sanger sequencing.

Phenotypic and Ara-C resistance analyses. For cytologic analysis, cells were cytospinned at $700 \mathrm{x} \mathrm{g}$ for $3 \mathrm{~min}$ or smeared, air-dried, and stained with Liu reagents (Tonyar Biotech, Inc.) at room temperature. For immunophenotypic analysis, cells were stained at $4^{\circ} \mathrm{C}$ for 15 min with phycoerythrin-macrophage-1 antigen (Mac-1), phycoerythrin-CD115 and allophycocyanine-Ki-67 antibodies (cat. nos. RM2804-3, 12-1152-82 and 17-5698-82; eBioscience; Thermo Fisher) followed by flow cytometric analysis using FACSCanto II Cell Analyzer and FACSDiva software version 5.0 (BD Biosciences). For cell proliferation analysis, cells were assessed at indicated time points using Cell Counting Kit- 8 (CCK-8/WST-8; Dojindo Molecular Technologies, Inc.) according to the manufacturer's instructions. To determine Ara-C resistance, cells were cultured in RPMI- 1640 complete medium and a gradient concentration of Ara-C $(0,128,320$, $640,1,600,3,200,16,000$ or $40,000 \mathrm{ng} / \mathrm{ml}$ ) for $24 \mathrm{~h}$. Cell viability was measured using CCK-8 (incubation time $2 \mathrm{~h}$ ). To determine apoptotic cell rate, cells were treated with Ara-C ( 0 or $160 \mathrm{ng} / \mathrm{ml}$ ) for $24 \mathrm{~h}$, followed by Annexin-V/propidium iodide (Sigma-Aldrich; Merck KGaA) staining in the dark and flow cytometric analysis using a FACSCanto II Cell Analyzer and FACSDiva software version 5.0 (BD Biosciences).

Competitive engraftment and clonal expansion assay. The competitive engraftment and clonal expansion assay was described previously $(22,33)$. Briefly, paired cells (AK3G-shV vs. AK3G-shH11 and 12G-V vs. 12G-H11) were mixed at a ratio of $1: 1$ and then i.p. injected into B6 mice $\left(1 \times 10^{6}\right.$ cells/mouse $)$. The mice were sacrificed at days 43 and 57. Mice BMs and spleens were collected and used to extract genomic DNA. To amplify 300-bp DNA fragments of the pMSCVpuro vector (from $12 \mathrm{G}-\mathrm{V}$ ) or the region spanning the pMSCVpuro-Hoxall junction (from 12G-H11) from genomic DNA, PCR was performed using the following 
primers: MSCV 5' primer 5'-CCCTTGAACCTCCTCGTT CAGCC-3' in combination with MSCV 3' primer 5'-GAG ACGTGCTACTTCCATTTGTC-3' or Hoxall primer 5'-GAG TAGCAGTGGGCCAGATTGC-3'. The PCR products were sequenced using MSCV 5' primer, and the peak height of the 62 th nucleotide (C for $12 \mathrm{G}-\mathrm{H} 11$ and $\mathrm{T}$ for $12 \mathrm{G}-\mathrm{V}$ ) was measured. The $(\mathrm{C} / \mathrm{C}+\mathrm{T})$ peak height ratio was converted to the cell ratio (12G-H11/12G-H11+12G-V) by aligning to the standard curve. The standard curve was generated by assessing the relationship between nucleotide peak height ratios and cell ratios from cell mixtures with mixed paired cells in ratios of 10:0, 9:1, 8:2, 7:3, 6:4, 5:5, 4:6, 3:7, 2:8, 1:9 and 0:10.

Ethics statement. All animal experiments were performed in accordance with the Guide for the Care and Use of Laboratory Animals published by National Institutes of Health (publication no. 85-23, revised 1996) and were carried out according to the protocol approved by the Animal Research Committee of Chang Gung Memorial Hospital (IACUC No. 2014092403; Taoyuan, Taiwan). Human sample collection was conducted in accordance with the Declaration of Helsinki and was approved by the Chang Gung Memorial Hospital Research Ethics Committee (IRB No. 96-1748B). Written informed consent was obtained from all patients. For patients under the age of 18 , consent/permission was obtained from the parents/guardians.

Statistical analysis. Statistical analyses were performed using SPSS software version 20.0 (SPSS, Inc). The statistical significance of differences in gene expression levels of the two groups was compared using a Mann-Whitney test. Survival analysis was conducted according to the Kaplan-Meier method, and differences in survival were assessed using the log-rank test. Drug sensitivity was compared using an unpaired two-sample Student's t-test. $\mathrm{P}<0.05$ was considered to indicate a statistically significant difference.

\section{Results}

Identification of DEGs and GO enrichment analysis. Our previous studies demonstrated that mice transplanted with AK2G, AK3G or APm-1 had shorter survival than those transplanted with the $12 \mathrm{G}$ and $\mathrm{APw}-1$, respectively $(21,22,26)$. Moreover, APm- 1 cells have been reported to be more resistant to daunorubicin, but more sensitive to Ara-C than APw-1 cells (22). To identify genes involved in the acceleration of leukemia development and drug resistance by cooperation of $M L L / A F 10$ with $R A S$ signaling mutations, transcriptomic profiling were compared between 6 paired $M L L / A F 10(O M-L Z)$ leukemia cells harboring wild-type and oncogenic KRAS (12G vs. AK3G, $12 \mathrm{G}$ vs. AK2G and AKw1G vs. AK3G) or PTPN11 (APw-1 vs. APm-1) using cDNA microarray data. A total of 23 DEGs (seven upregulated and 16 downregulated) with $>1.5$-fold-change were identified in AK3G and APm-1 cell lines compared with $12 \mathrm{G} / \mathrm{AKw} 1 \mathrm{G}$ and $\mathrm{APw}-1$ cells, respectively (Fig. 1B). The GO terms 'BP' and 'MF' enriched in the 23 DEGs included 'innate immune response', 'immune system process', 'actin filament binding', 'cellular response to interferon-alpha', 'sequence-specific DNA' and 'regulation of gene expression' (Fig. 1C). Among these enriched GO terms, three terms ('innate immune response', 'immune system process' and 'cellular response to interferon-alpha') are related to the immune response; 'actin filament binding' is related to cell protrusion and migration, while the last two terms ('sequence-specific DNA' and 'regulation of gene expression') are related to the regulation of gene expression by transcription factors.

Expression of transcription factors in patients with $A M L$ with MLL-t and/or RAS signaling mutations. A total of four genes, Hoxall, PR domain zinc finger protein 5 (Prdm5), Iroquois-class homeodomain protein IRX-5 (Irx5) and homeobox protein PKNOX2 (Pknox2), were mapped to the GO 'BP' term 'sequence-specific DNA'; other than Pknox2, these genes were upregulated in the AK3G and APm- 1 cell lines according to cDNA microarray data (Fig. 1B). To investigate whether these genes were upregulated in the patients with AML with $M L L$-t, KRAS or PTPN11 mutations, meta-analysis was performed using datasets deposited by Valk (285 AML cases), Wouters (503 AML cases), Haferlach (542 AML cases), and Balgobind ( 237 childhood AML cases) in the Oncomine ${ }^{\mathrm{TM}}$ clinical research data repository for gene expression change. The results revealed that two (Balgobind and Haferlach) of the four AML series showed significant differences $(\mathrm{P}<0.01)$ in HOXA11 expression for patients with AML with or without $M L L$-t (11q23-r) using reporter ID 208493 (Fig. 2A). One (Valk) of the two AML series showed significant differences $(\mathrm{P}<0.05)$ in HOXA11 expression for patients with AML with or without KRAS activating mutations (KRASm) (Fig. 2B). In the Balgobind series, there was a trend of higher HOXAll expression in patients with AML with PTPN11 mutations than those without PTPN11 mutations, but the difference was not statistically significant (Fig. 2C). This is probably due to the low case numbers $(n=5)$. On the other hand, there were no significant differences in IRX5 and PRDM5 expression in leukemia cells of patients with AML with or without $M L L-\mathrm{t}$, KRAS or PTPN11 mutations (Fig. S1A-F).

HOXA11 expression was also determined in the patients with $M L L-t$ AML included in the present study. The results showed that $M L L / A F 10^{+}$patients with AML with KRAS or PTPNII mutations had higher levels of HOXAll expression than patients with wild-type KRAS and PTPN11 genes (Fig. 2D). Similarly, $M L L / A F 9^{+}$patients with AML with $K R A S$ mutations expressed higher levels of HOXA11 compared with the patient with wild-type $K R A S$ (Fig. 2E). These results supported that cooperation of $M L L-\mathrm{t}$ with oncogenic KRAS or PTPNII mutation induces HOXA11 expression in patients with AML.

Differential expression of Hoxa genes in mouse MLL/AF10 leukemia cells with different RAS signaling mutations. Based on the cDNA microarray data, HoxalO and Hoxall were upregulated (>1.5-fold) in AK3G compared with 12G cells (Fig. 3A). Compared with AKw1G, AK3G cells had higher expression levels (>1.5-fold) of Hoxa5, Hoxa6, Hoxal0 and Hoxall (Fig. 3B). Compared with APw-1, only Hoxall was upregulated in APm-1 cells (Fig. 3C). These data suggested that KRAS and PTPN11 mutations had overlapping, but not exactly the same effects on Hoxa gene expression in MLL/AF10 leukemia cells. Conversely, no significant differences in the expression levels of Hoxb, Hoxc, Hoxd and Meisl genes were observed in these three pairs of mouse leukemia cell lines (Fig. 3A-C). 
A
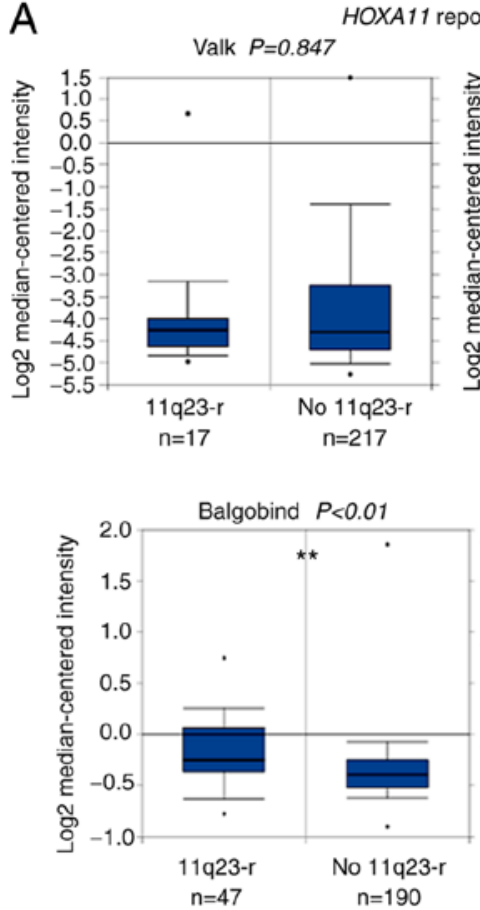
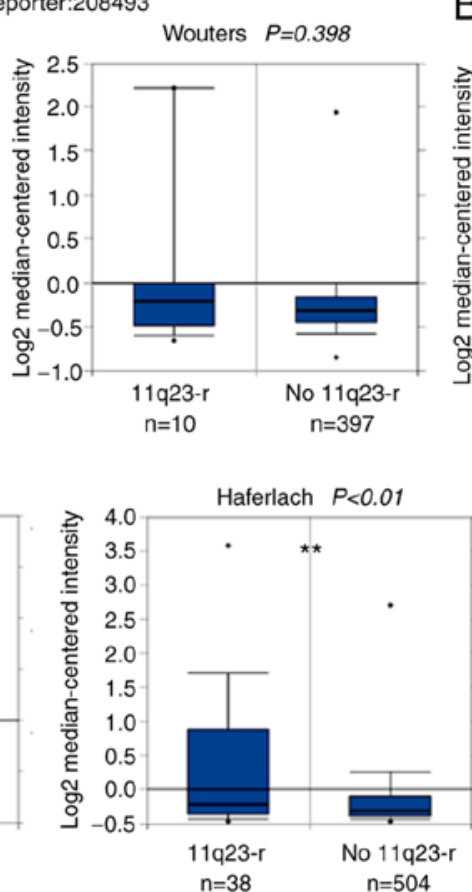

HOXA11

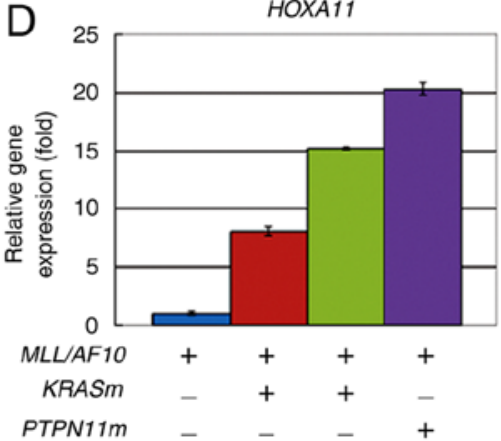

B
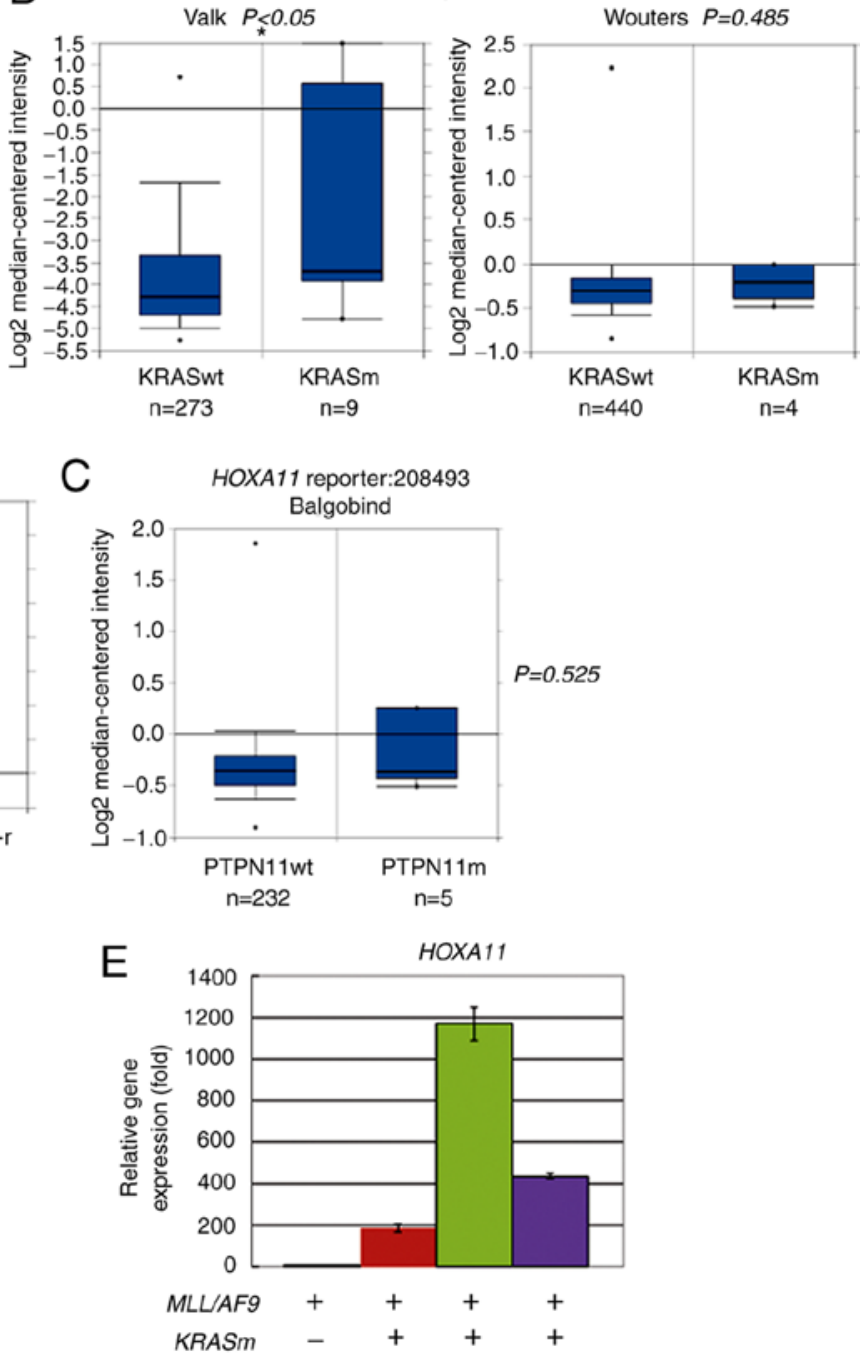

Figure 2. Overexpression of HOXA11 in AML carrying MLL-t, oncogenic KRAS or PTPN11 mutations. (A-C) Meta-analysis of HOXA11 expression in AML using leukemia database deposited by Valk $(\mathrm{n}=285)$, Wouters $(\mathrm{n}=503)$, Haferlach $(\mathrm{n}=542)$ and Balgobind ( $\mathrm{n}=237$, childhood AML) in Oncomine ${ }^{\mathrm{TM}}$. Box plots are data of HOXA11 (reporter ID. 208493) based on cDNA microarray data in patients with AML (A) with 11q23-r or No 11q23-r, (B) patients with KRASm or KRASwt, and (C) patients with PTPN11m or PTPN11wt. Numbers listed at the bottom are case numbers. Center line in box plot represents median value, box limits are 10 and 90th percentiles, and dots represent minimum and maximum values. P-values were determined using an unpaired two-sample Student's t-test. ${ }^{*} \mathrm{P}<0.05$ and ${ }^{* *} \mathrm{P}<0.01$. (D and E) Reverse transcription-quantitative PCR analyses were performed to determine relative $H O X A 11$ expression level in patients with AML carrying (D) $M L L / A F 10$ or (E) $M L L / A F 9$ with or without oncogenic KRASm or PTPN11m mutations. Assays were performed in triplicate and data are representative of three independent experiments. Error bars indicate SD of mean. AML, acute myeloid leukemia; MLL, lysine methyltransferase 2A; $M L L$-t, $M L L$ translocations; PTPN11, tyrosine-protein phosphatase non-receptor type 11; 11q23-r, $M L L$ rearrangements; No 11q23-r, wild-type $M L L$; KRASm, oncogenic KRAS mutations; KRASwt, wild-type KRAS; PTPN11m, oncogenic PTPN11 mutations; PTPN11wt, wild-type PTPN11.

RT-qPCR and western blotting confirmed that the transcriptional and translational levels of Hoxall were increased in $M L L / A F 10(O M-L Z)$ mouse leukemia cell lines harboring KRAS mutation (AK2G, AK3G) and PTPN11 mutation (APm-1) compared with cells harboring wild-type KRAS (12G, AKw1G) or wild-type PTPN11 (APw-1) (Fig. 3D and E).

Role of Hoxall in survival. To determine the role of Hoxall in the leukemogenesis of $M L L / A F 10$ leukemia cells harboring $R A S$ pathway mutations, two lentivirus-based shRNAs that targeted mouse Hoxall gene (shH11-1 and shH11-2) were stably transduced into APm- 1 cells. RT-qPCR analysis of the Hoxal1 knockdown APm-1 (APm-1-shH11-1 and APm-1-shH11-2) cell lines showed that the expression levels of Hoxall were reduced to 86 and $46 \%$, respectively, compared with the control cell line (APm-1-shV) (Fig. 4A). By combining the fold changes in Hoxall between APw-1 and APm-1 and between APm-1-shV and APm-1-shH11-1, the expression level of Hoxall in APm-1-shH11-2 cells was estimated to be 2.4-fold higher than in APw-1 cells (Figs. 3D and 4A). Western blot analysis further confirmed that the protein levels of Hoxall were decreased in APm-1-shH11 cell lines compared with the APm-1-shV control cell line (Fig. 3E). Mice i.p. injected with APm-1-shH11-2 cells, which had lower Hoxall expression, had significantly longer survival than those injected with APm-1-shV cells (median 64 days vs. 50 days; $\mathrm{P}<0.01$; Fig. $4 \mathrm{~B}$ and Table I). To further support the role of Hoxall in the survival of leukemic mice, Hoxal1 overexpressed $12 \mathrm{G}$ cells (12G-H11-1 and 12G-H11-2) were generated by transduction of retrovirus-based full-length 

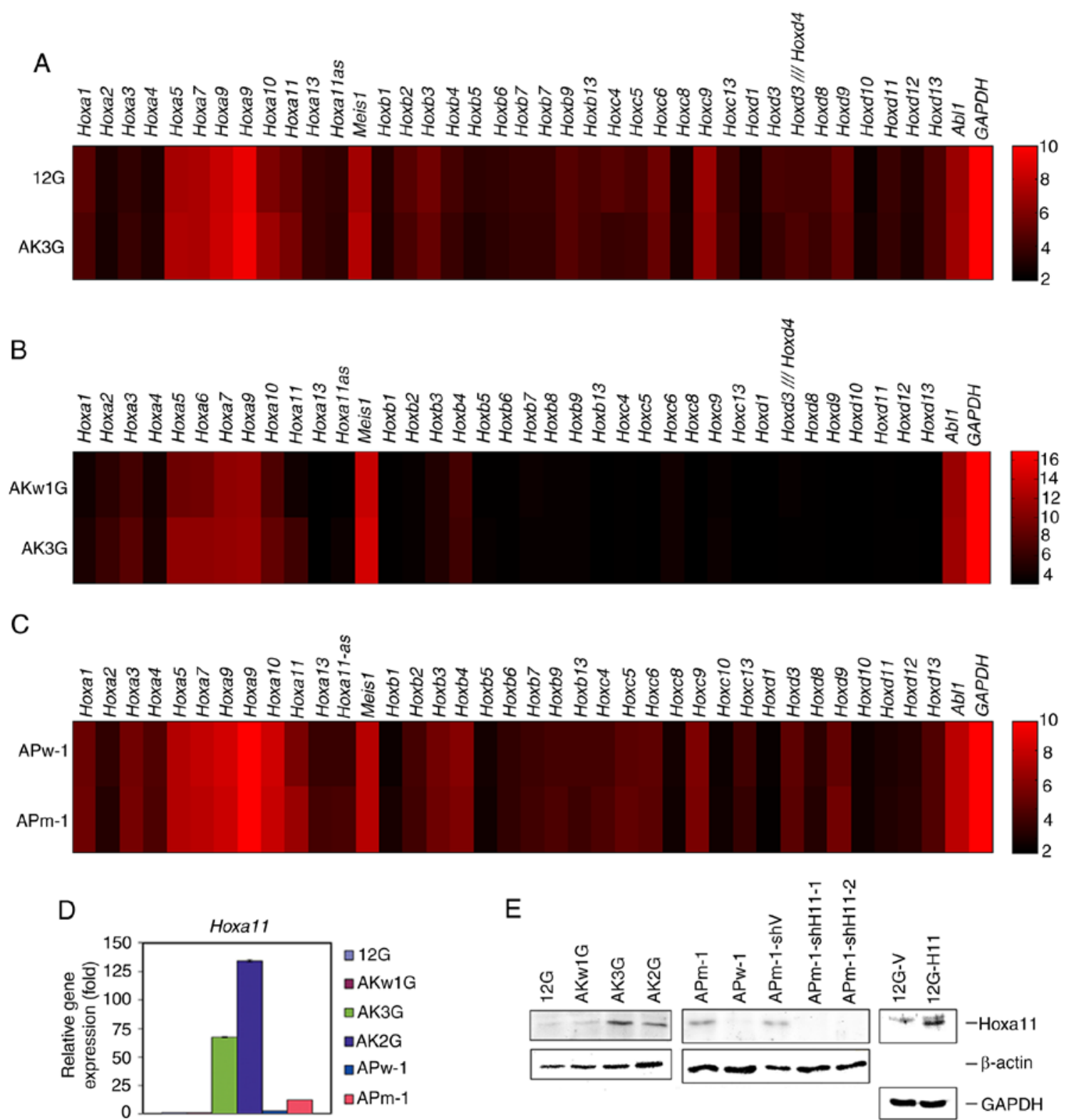

Figure 3. Expression levels of Hox clusters A, B, C, D and Meis1 genes in paired MLL/AF10 leukemia cells harboring wild-type or oncogenic RAS pathway mutations. (A-C) Heat maps of genes based on cDNA microarray data between paired cell lines: (A) 12G and AK3G, (B) AKw1G and AK3G, and (C) APw-1 and APm-1. Raw values are log2-transformed. Red and black indicate high and low levels of gene expression, respectively. Color bar depicts log2-transformed value of genes. (D) Reverse transcription-quantitative PCR analysis was performed to determine the level of Hoxall expression in 12G, AKw1G, AK3G, AK2G, APw-1 and APm-1 cells. Assays were performed in triplicate and data are representative of three independent experiments. Error bars indicate SD. (E) Western blotting was performed to determine the protein level of Hoxa11 in cells of the different cell lines. The detection of $\beta$-actin and Gapdh served as a loading control for immunoblot analysis. MLL, lysine methyltransferase $2 \mathrm{~A} ; 12 \mathrm{G}$, cells with $M L L / A F 10(O M-L Z)$ alone; AK3G, cells with $M L L / A F 10(O M-L Z)$ and oncogenic KRASG12C; AKw1G, cells with MLL/AF10(OM-LZ) and wild-type KRAS; APw-1, cells with MLL/AF1O(OM-LZ) and wild-type PTPN11; APm-1, cells with MLL/AF10(OM-LZ) and oncogenic PTPN11G503A; PTPN11, tyrosine-protein phosphatase non-receptor type 11.

Hoxall gene into $12 \mathrm{G}$ cells. The $12 \mathrm{G}$ cells transduced with empty retroviruses (12G-V1 and $12 \mathrm{G}-\mathrm{V} 3)$ served as controls. Overexpression of Hoxall in the 12G-H11-1 and 12G-H11-2 cell lines compared with the control 12G-V1 and 12G-V3 cell lines was confirmed by western blotting and RT-qPCR (Figs. 3E and 4C). BM transplantation assay data showed that $12 \mathrm{G}-\mathrm{H} 11-1$ mice had significantly shorter survival than control 12G-V1 mice (median 76 days vs. 91 days; $\mathrm{P}<0.001$; Fig. 4D and Table I). These results indicated that Hoxall plays a critical role in the survival of leukemic mice induced by $M L L / A F 10$ leukemia cells and $M L L / A F 10$ cells harboring activating PTPN11 mutation.
Compared with the control cell lines, Hoxal1-knockdown APm-1 cells and Hoxall-overexpression 12G cells had similar blast-like morphology (Fig. S2A and C, left column), similar percentages of CD $115^{+}$cells (Fig. S2A and $\mathrm{C}$, right column), and similar cell proliferation rates in vitro (Fig. S2B and D). These results suggested that the role of Hoxall in the acceleration of disease progression in the leukemia mice was not caused by changes in differentiation potential or proliferation rate of the $M L L / A F 10$ leukemia cells. Flow cytometry analysis of Ki-67, which detects proliferating cells, was performed on the leukemia cells $\left(\mathrm{Mac1}^{+}\right)$obtained from BM of 12G-V1 and 12G-H11-1 
A

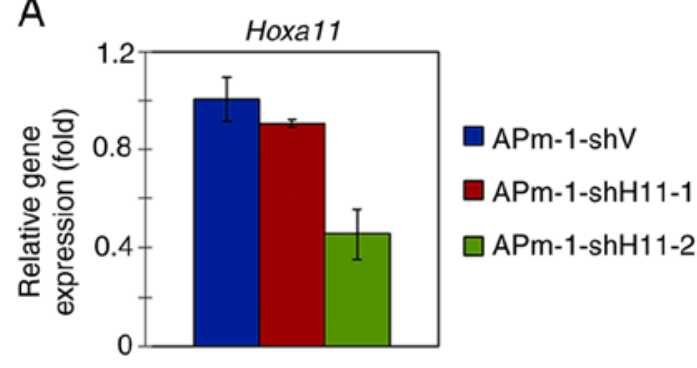

C

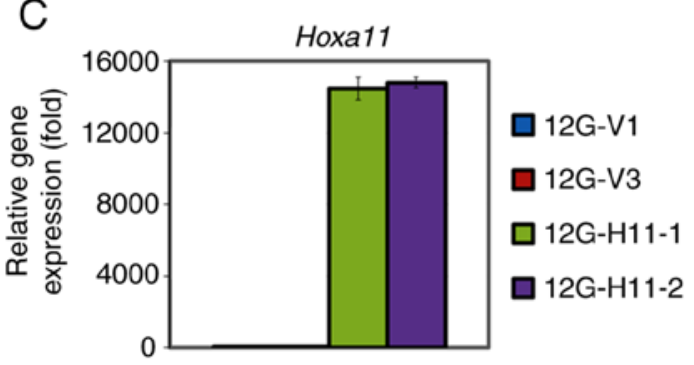

E

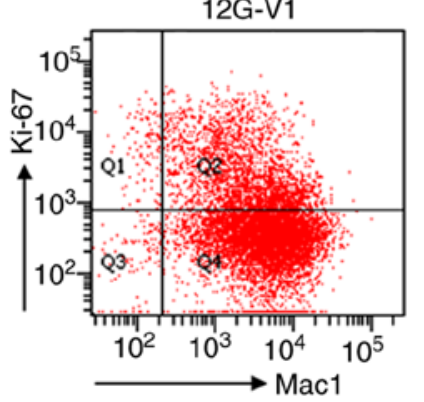

12G-H11-1

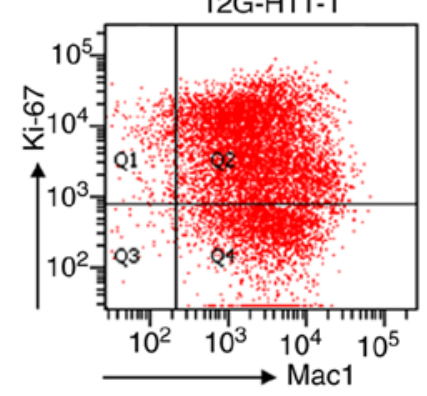

B
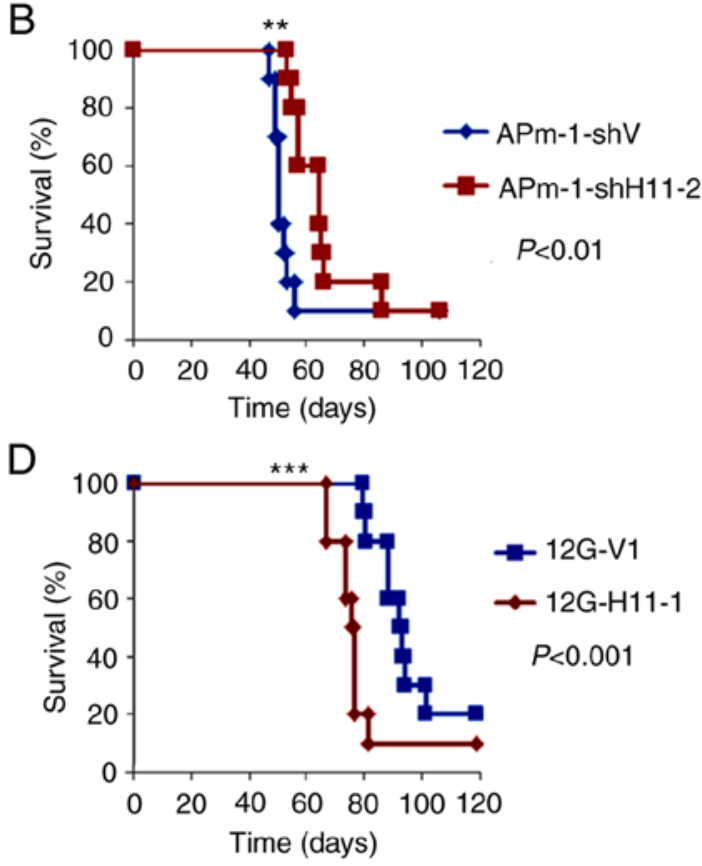

$\mathrm{F}$

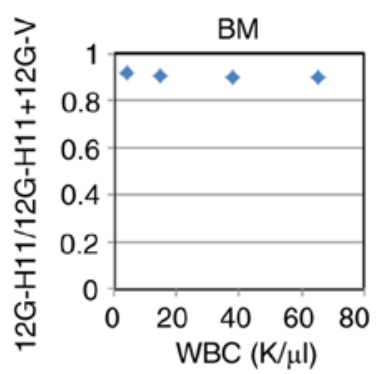

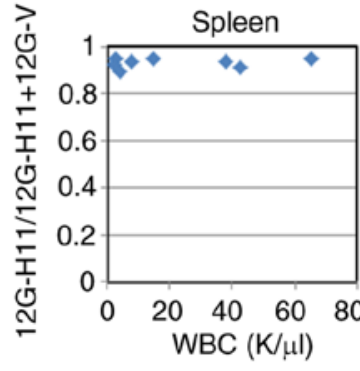

Figure 4. Expression of Hoxal1 affects survival of MLL/AF10 leukemia mice. (A and C) Reverse transcription-quantitative PCR analyses were performed to determine Hoxal1 expression in (A) Hoxal1-knockdown APm-1 (APm-1-shH11-1, APm-1-shH11-2) and control (APm-1-shV) cell lines, or in (C) Hoxa11-overexpression 12G (12G-H11-1, 12G-H11-2) and control (12G-V1 and 12G-V3) cell lines. Assays were performed in triplicate and data shown are representative of three independent experiments. Error bars indicate SD. (B and D) Survival curves of mice i.p. injected with (B) APm-1-shV or APm-1-shH11-2, and (D) $12 \mathrm{G}-\mathrm{V} 1$ or $12 \mathrm{G}-\mathrm{H} 11-1$ cells. Survival analysis was conducted according to the Kaplan-Meier method. ${ }^{* *} \mathrm{P}<0.01$ and ${ }^{* * *} \mathrm{P}<0.001$ vs. APm-1-shV or 12G-V1 cells. (E) Flow cytometry analyses was performed to determine Ki-67 and Mac-1 expression in the BM cells obtained from 12G-V1 and 12G-H11-1 leukemia mice at moribund stage. Data shown are representative of three mice with similar results. (F) Competitive engraftment and clonal expansion ability between 12G-V1 and 12G-H11-1 cells in vivo. 12G-V1 and 12G-H11-1 cells were mixed in a 1:1 ratio and i.p. injected into recipient mice (n=9). The mice were sacrificed at 43 and 57 days post-transplantation ( $\mathrm{n}=2$ and $\mathrm{n}=7$, respectively). Allele burden of the 12G-H11-1 clone in BM (left column) or spleen (right column) was determined by PCR-DNA sequencing. MLL, lysine methyltransferase 2A; APm-1, cells with $M L L / A F 10(O M-L Z)$ and oncogenic PTPN11G503A; sh, short hairpin RNA; 12G, cells with $M L L / A F 10(O M-L Z)$ alone; i.p. intraperitoneally; Mac-1, macrophage-1 antigen; BM, bone marrow; PTPN11, tyrosine-protein phosphatase non-receptor type 11 .

mice. The results showed that $12 \mathrm{G}-\mathrm{H} 11-1$ cells were more actively proliferating in vivo (Fig. 4E). To investigate whether Hoxall enhanced growth advantage of MLL/AF10 leukemia cells in vivo, competitive engraftment and clonal expansion assays were performed. Compared with the control 12G-V1, 12G-H11-1 cells were more competitive to engraft to and expand in BM and spleen (Figs. 4F and S3A-C). These results suggested that Hoxall promotes disease progression, at least partly, by promoting leukemia cell recruitment and proliferation in their niche.

On the other hand, AK3G cells were also stably transduced with the two lentivirus-based shRNAs. Although the knockdown efficiencies of the shRNAs were significant (Fig. S4), the Hoxal1 expression levels in AK3G-shH11-2 cells were estimated to be 16.9-fold and 21.7-fold higher than that of $12 \mathrm{G}$ and AKw1G cells, respectively, based on the combined RT-qPCR results (Figs. 3D and S4). Moreover, AK3G cells had higher expression levels of Hoxa5, Hoxa6 and Hoxal0 compared with AKw1G (Fig. 3B). Due to the high Hoxall expression levels and the possible complementary effect of the Hoxa5-a10 genes (11), no further assays were performed to assess survival and drug sensitivity of AK3G-shV and AK3G-shH11 cells in this study.

Role of Hoxall in Ara-C resistance. To determine whether Hoxall affects Ara-C resistance of $M L L / A F 10$ leukemia cells harboring PTPN11 $1^{G 503 A}$, APm-1-shV and APm-1-shH11-2 cells were treated with a gradient concentration of Ara-C for $24 \mathrm{~h}$. Compared with APm-1-shV cells, APm-1-shH11-2 cells exhibited significantly higher viability at Ara-C concentrations between 128-1,600 ng/ml ( $\mathrm{P}<0.01$; Fig. 5A). The median lethal dose (LD50) of Ara-C for APm-1-shV and APm-1-shH11-2 
Table I. Phenotypic characteristics of the mice transplanted with the Hoxall-knockdown APm-1 and Hoxall-overexpression $12 \mathrm{G}$ leukemia cells.

A, Hoxa11-knockdown APm-1 cells

\begin{tabular}{|c|c|c|c|}
\hline Features & APm-1-shV & APm1-shH11 & P-value \\
\hline Number of mice, $n$ & 10.0 & 10.0 & \\
\hline Survival median, days, $\mathrm{n}(\text { range })^{\mathrm{a}}$ & $50.0(47.0-56.0)$ & $64.0(53.0-86.0)$ & 0.00543 \\
\hline $\mathrm{WBC}, 1 \times 10^{9} / \mathrm{ml}$, median (range) ${ }^{\mathrm{a}}$ & $64.2(58.0-97.6)$ & $85.5(41.2-207.2)$ & 0.25160 \\
\hline Anemia, n/n $(\%)^{\mathrm{b}}$ & $4 / 4(100.0)$ & $6 / 6(100.0)$ & 1.00000 \\
\hline Thrombocytopenia, n/n $(\%)^{\mathrm{b}}$ & $1 / 4(25.0)$ & $3 / 6(50.0)$ & 0.57100 \\
\hline Ascites, $\mathrm{n} / \mathrm{n}(\%)^{\mathrm{b}}$ & $3 / 8(37.5)$ & $0 / 8(0.0)$ & 0.20000 \\
\hline Hepatosplenomegaly, n/n (\%) & $8 / 8(100.0)$ & $9 / 9(100.0)$ & 1.00000 \\
\hline Myeloid sarcoma, n/n (\%) & $1 / 8(12.5)$ & $1 / 8(12.5)$ & 1.00000 \\
\hline
\end{tabular}

B, Hoxall-overexpression $12 \mathrm{G}$ cells

\begin{tabular}{|c|c|c|c|}
\hline Features & $12 \mathrm{G}-\mathrm{V}$ & 12G-H11 & P-value \\
\hline Number of mice, $\mathrm{n}$ & 10.0 & 10.0 & \\
\hline Survival median, days, $\mathrm{n}(\text { range })^{\mathrm{a}}$ & $91.0(80.0-102.0)$ & $76.0(67.0-82.0)$ & 0.00023 \\
\hline WBC, $1 \times 10^{9} / \mathrm{ml}$, median $(\text { range })^{\mathrm{a}}$ & $151.6(61.1-354.6)$ & $58.8(23.3-149.1)$ & 0.07290 \\
\hline Anemia, n/n $(\%)^{\mathrm{b}}$ & $1 / 8(12.5)$ & $2 / 5(40.0)$ & 0.51049 \\
\hline Thrombocytopenia, $\mathrm{n} / \mathrm{n}(\%)^{\mathrm{b}}$ & $2 / 8(25.0)$ & 4/7 (57.1) & 0.31469 \\
\hline Ascites, $\mathrm{n} / \mathrm{n}(\%)^{\mathrm{b}}$ & $2 / 9(22.2)$ & $0 / 8(0.0)$ & 0.47059 \\
\hline Hepatosplenomegaly, n/n (\%) & $8 / 8(100.0)$ & $9 / 9(100.0)$ & 1.00000 \\
\hline Myeloid sarcoma, n/n (\%) & $1 / 9(22.2)$ & $0 / 8(0.0)$ & 1.00000 \\
\hline
\end{tabular}

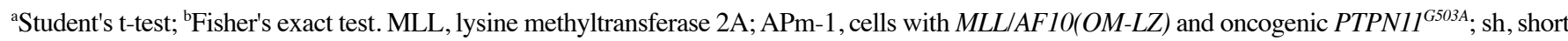
hairpin RNA; 12G, cells with $M L L / A F 10(O M-L Z)$ alone; PTPN11, tyrosine-protein phosphatase non-receptor type 11; WBC, white blood cells .

cells was 235 and $315 \mathrm{ng} / \mathrm{ml}$, respectively. By contrast, Hoxal1-overexpressing 12G-H11-1 cells showed significantly lower cell viabilities at all tested concentrations of Ara-C $(\mathrm{P}<0.005$; Fig. 5B). The LD50 of Ara-C for $12 \mathrm{G}-\mathrm{V}$ and 12G-H11 cells was 459 and $224 \mathrm{ng} / \mathrm{ml}$, respectively. Further studies revealed that the apoptosis rate of APm-1-shH11-2 cells was lower than that of APm-1-shV cells before (17.6 vs. $25.4 \%$, respectively) and after (41.3 vs. $45.2 \%$, respectively) Ara-C treatment $(160 \mathrm{ng} / \mathrm{ml})$ for $24 \mathrm{~h}(\mathrm{Fig} .5 \mathrm{C})$. The apoptosis rate of $12 \mathrm{G}-\mathrm{H} 11-1$ cells was higher than that of $12 \mathrm{G}-\mathrm{V} 1$ cells before (30.5 vs. $14.9 \%$, respectively) and after Ara-C treatment (57.8 vs. 37.2\%, respectively) (Fig. 5D). These results indicated that cooperation of $M L L / A F 10$ with $P T P N 11^{G 503 A}$ upregulated Hoxa11, which in turn increased cell apoptosis and rendered cells more sensitive to Ara-C.

As Hoxall encodes a DNA-binding transcription factor and affects apoptosis of leukemia cells, the present study next determined the expression levels of three apoptosis-related genes in Hoxal1-knockdown APm-1 and Hoxal1-overexpression $12 \mathrm{G}$ cells using RT-qPCR analysis. It was found that the silencing of Hoxall significantly increased the expression levels of Nfkbia, Rela and Trp53, whereas the overexpression of Hoxall significantly reduced the expression level of these genes (Fig. 5E). These results suggested that Hoxall induces cell apoptosis, at least partly, via regulation of apoptosis-related gene expression.
To determine whether the expression of HOXA11 in leukemia cells of patients with AML was associated with chemotherapy drug sensitivity, a meta-analysis was performed using a data set deposited by Heuser consisting of 33 AML cases enrolled in the AML-SHG 01/99 trial, in the Oncomine $^{\mathrm{TM}}$ clinical research data repository for gene expression change (34). The results of this analysis showed that responders of chemotherapy or AML induction/consolidation had higher $H O X A 11$ expression levels than non-responders using reporter AA598674 (Fig. 5F-a and F-b). Collectively, these findings indicated that patients with AML with higher HOXA11 expression are associated with an improved response to chemotherapy with Ara-C.

\section{Discussion}

The present study compared transcriptomic profiling between mouse $M L L / A F 10$ leukemia cells harboring wild-type and activating KRAS or PTPN11, and found that Hoxa7-Hoxa10 were expressed in all $M L L / A F 10$ leukemia cell lines, whereas Hoxall was only expressed in MLL/AFlO leukemia cells with activating KRAS or PTPN11 mutations (Fig. 3A-C). Furthermore, a meta-analysis using microarray datasets deposited in Oncomine ${ }^{\mathrm{TM}}$ and an analysis of our clinical samples indicated that $H O X A 11$ is upregulated in MLL-t AML with $R A S$ signaling mutations. As $29.4 \sim 45.8 \%$ of cases with $M L L-\mathrm{t}$ 

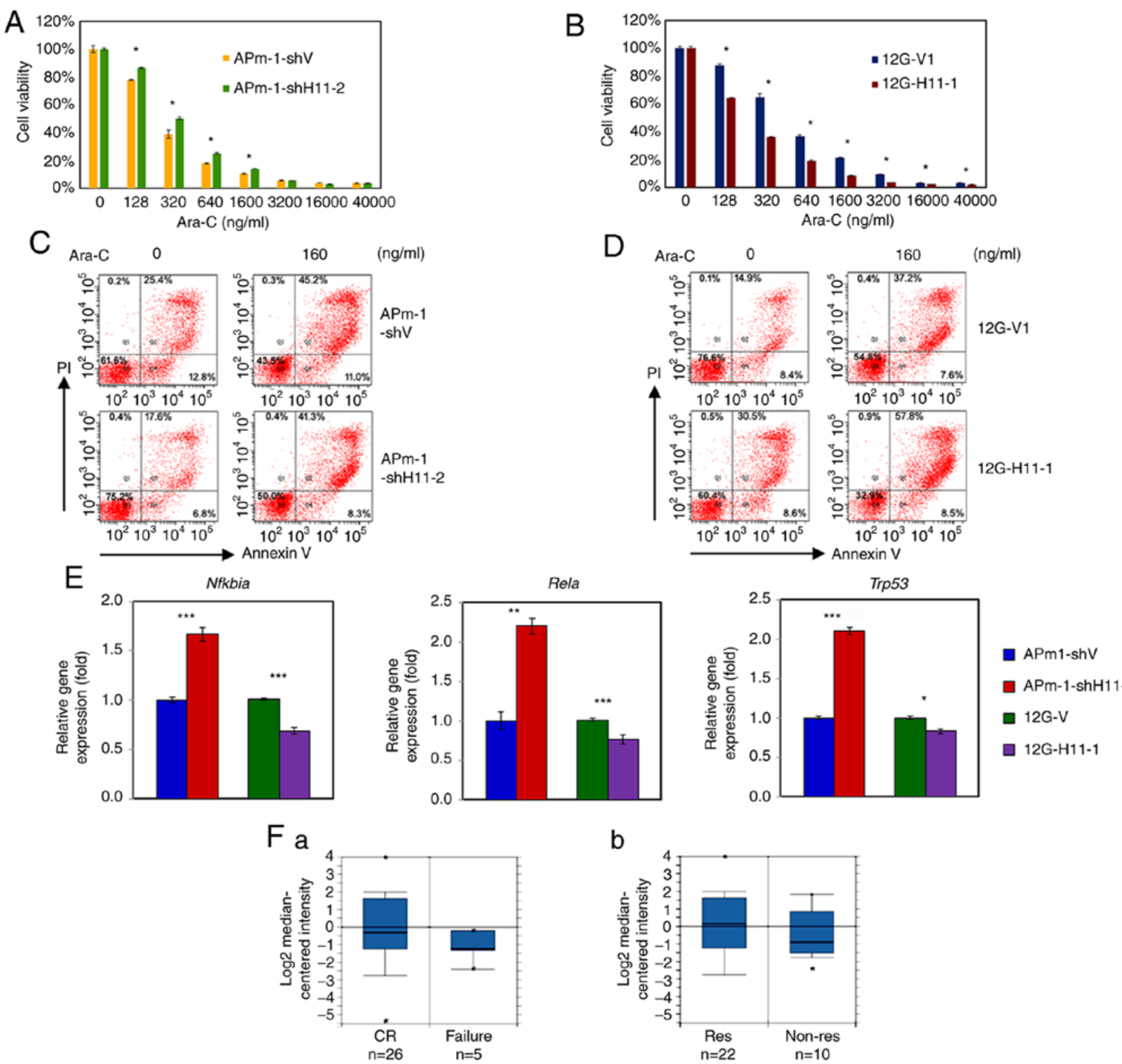

Figure 5. Hoxal1 expression affects chemotherapy drug resistance. (A-D) Viability assays of (A and C) Hoxal1-knockdown APm-1 (APm-1-shH11-2) and control (APm-1-shV) cell lines or (B and D) Hoxal1-overexpression 12G (12G-H11-1) and control (12G-V1) cell lines treated with Ara-C at indicated concentrations for $24 \mathrm{~h}$. The viability of leukemia cells was determined by a Cell Counting Kit-8 assay. Assays were performed in triplicate and data are representative of three independent experiments. The error bars indicate SD, and P-values were determined with an unpaired two-sample Student's t-test. (E) The expression levels of the apoptosis-related genes, Nfkbia, Rela and Trp53 in Hoxa11-knockdown or Hoxa11-overexpression MLL/AF10 leukemia cells were evaluated via reverse transcription-quantitative PCR. ${ }^{*} \mathrm{P}<0.05,{ }^{* *} \mathrm{P}<0.01,{ }^{* * *} \mathrm{P}<0.001$ vs. APm-1-shV or $12 \mathrm{G}-\mathrm{V} 1$ cells. (F) Meta-analysis of HOXA11 expression in AML using a leukemia database deposited by Heuser $(n=33)$ in Oncomine ${ }^{\mathrm{TM}}$. Box plots are the HOXA11 (reporter ID. AA598674) expression levels based on cDNA microarray data in patients with AML grouped by (F-a) chemotherapy responsiveness and (F-b) AML induction/consolidation response status. Numbers listed at the bottom are the number of cases. The center line in the box plot represents the median, the box limits indicate the 10 and 90th percentiles, and dots represent minimum and maximum values. MLL, lysine methyltransferase 2A; APm-1, cells with MLL/AF10(OM-LZ) and oncogenic PTPN11G503A; sh, short hairpin RNA; 12G, cells with MLL/AF1O(OM-LZ) alone; PTPN11, tyrosine-protein phosphatase non-receptor type 11; Ara-C, cytarabine; $N f k b i a$, NF- $\mathrm{BB}$ inhibitor $\alpha$; Rela, transcription factor p65; Trp53, transformation-related protein p53; AML, acute myeloid leukemia; CR, complete remission; Res, responder; Non-Res, non-responder.

AML harbor $N-/ K-R A S$ or PTPN11 mutations, this finding may partly explain why HOXAll expression is less frequently reported in $M L L$-t AML $(12,13)$.

Data obtained from BM transplantation assay using Hoxal1-knockdown or Hoxal1-overexpression MLL/AF10 leukemia cells revealed that the expression levels of Hoxall in leukemia cells was associated with the survival of recipient mice. It was also demonstrated that Hoxall overexpression promoted $M L L / A F 10(O M-L Z)$ leukemia cells to engraft and proliferate in $\mathrm{BM}$ and spleen (Fig. 4E and F). A similar observation was reported by Sun et al (35), which showed that HOXA11 overexpression promoted cell proliferation and migration, and reduced cell apoptosis in breast cancer.

Based on the present study analyses of in vitro cytotoxicity and apoptosis rate of Hoxall-knockdown and Hoxall-overexpression MLL/AF10(OM-LZ) leukemia cells showed that Hoxall expression was associated with Ara-C sensitivity and apoptotic cell rate (Fig. 5A-D). In 
addition, gene expression analysis revealed that Hoxall induced apoptosis, at least partly, by regulating the expression of apoptosis-related genes, including Nfkbia, Rela and Trp53 (Fig. 5E), which is in line with previous findings by Guo et al (36), which established Ara-C-resistant human AML OCI-AML2 cell lines. Based on a comparative transcriptomic analysis, they identified $H O X A 11$ as a DEG between resistant and parent cell lines, and further determined that $H O X A 11$ promoted Ara-C sensitivity and apoptosis in the cell line (36). Moreover, a meta-analysis of HOXA11 expression using microarray data deposited by Heuser in the present study supported the findings of an association between $H O X A 11$ expression and responsiveness of patients with AML treated with chemotherapy or on an AML induction/consolidation regimen (Fig. 5F). These data provided further support that HOXA11 expression in AML is predictive of an improved response to chemotherapy with Ara-C. The molecular mechanism of Hoxall in the regulation of apoptosis-related genes needs further characterization.

The role of $H O X A 11$ varies according to cancer type. Epigenetic inactivation of HOXA11 is a poor prognostic marker and contributes to disease progression in ovarian cancer, non-small cell lung cancer, gastric cancer, urothelial bladder cancer, glioblastoma, renal cell carcinoma and breast cancer (37-43). In these solid tumors, $H O X A 11$ acts as a functional tumor suppressor; however, the present results showed that upregulation of Hoxall accelerated leukemia development in MLL/AF10 cooperating $R A S$ pathway mutations, suggesting an oncogenic role of Hoxall rather than one of tumor suppression. Further investigation of downstream Hoxall targets and biological pathways will provide an improved understanding of the mechanism underlying the different roles of Hoxall in AML and solid tumors.

\section{Acknowledgements}

We would like to thank Mr. Jun-Wei Huang (Chang Gung Memorial Hospital, Taoyuan, Taiwan) and Mr. Chih-Shien Chuang (Chang Gung Memorial Hospital, Taoyuan, Taiwan) for their technical assistance in all the experiments.

\section{Funding}

This work was supported by grants from the Ministry of Science and Technology, Taiwan (grant no. 107-2320-B-182A013) and Chang Gung Memorial Hospital, Taiwan (grant nos. CMRPG3E0301-3, CMRPG3E1391-3, CMRPG3G1821 and CMRPG3J1331).

\section{Availability of data and materials}

The datasets used and/or analyzed during the current study are available from the corresponding author on reasonable request.

\section{Authors' contributions}

JFF was responsible for the conception and design of the present study, data analysis, funding acquisition and writing the original draft. LYS was responsible for providing the resources, analysis and interpretation of data, and wrote, reviewed and edited the manuscript. THY was responsible for data analysis, interpretation and discussion. JFF and LYS confirm the authenticity of all the raw data. All authors read and approved the final manuscript.

\section{Ethics approval and consent to participate}

All animal experiments were performed in accordance with the National Institutes of Health Guide for the Care and Use of Laboratory Animals and were carried out according to the protocol approved by the Animal Research Committee of Chang Gung Memorial Hospital (IACUC No. 2014092403; Taoyuan, Taiwan). Human sample collection was conducted in accordance with the Declaration of Helsinki and was approved by the Chang Gung Memorial Hospital Research Ethics Committee (IRB No. 96-1748B). Informed consent was obtained from all patients. For patients under the age of 18 , consent/permission was obtained from the parents/guardians.

\section{Patient consent for publication}

Not applicable.

\section{Competing interests}

The authors declare that they have no competing interests.

\section{References}

1. Meyer C, Hofmann J, Burmeister T, Gröger D, Park TS, Emerenciano M, Pombo de Oliveira M, Renneville A, Villarese P, Macintyre E, et al: The MLL recombinome of acute leukemias in 2013. Leukemia 27: 2165-2176, 2013.

2. Meyer C, Burmeister T, Gröger D, Tsaur G, Fechina L, Renneville A, Sutton R, Venn NC, Emerenciano M, Pombo-deOliveira MS, et al: The MLL recombinome of acute leukemias in 2017. Leukemia 32: 273-284, 2018.

3. Balgobind BV, Raimondi SC, Harbott J, Zimmermann M, Alonzo TA, Auvrignon A, Beverloo HB, Chang M, Creutzig U, Dworzak MN, et al: Novel prognostic subgroups in childhood 11q23/MLL-rearranged acute myeloid leukemia: Results of an international retrospective study. Blood 114: 2489-2496, 2009.

4. Grimwade D, Hills RK, Moorman AV, Walker H, Chatters S, Goldstone AH, Wheatley K, Harrison CJ, Burnett AK and National Cancer Research Institute Adult Leukaemia Working Group: Refinement of cytogenetic classification in acute myeloid leukemia: Determination of prognostic significance of rare recurring chromosomal abnormalities among 5876 younger adult patients treated in the United Kingdom Medical Research Council trials. Blood 116: 354-365, 2010.

5. Yu BD, Hess JL, Horning SE, Brown GA and Korsmeyer SJ: Altered Hox expression and segmental identity in Mll-mutant mice. Nature 378: 505-508, 1995.

6. Mohi MG, Williams IR, Dearolf CR, Chan G, Kutok JL, Cohen S, Morgan K, Boulton C, Shigematsu H, Keilhack H, et al: Prognostic, therapeutic, and mechanistic implications of a mouse model of leukemia evoked by Shp2 (PTPN11) mutations. Cancer Cell 7: 179-191, 2005.

7. Horton SJ, Grier DG, McGonigle GJ, Thompson A, Morrow M, De Silva I, Moulding DA, Kioussis D, Lappin TR, Brady HJ and Williams O: Continuous MLL-ENL expression is necessary to establish a 'Hox Code' and maintain immortalization of hematopoietic progenitor cells. Cancer Res 65: 9245-9252, 2005.

8. Suzuki A, Ito Y, Sashida G, Honda S, Katagiri T, Fujino T, Nakamura T and Ohyashiki K: t(7;11)(p15;p15) Chronic myeloid leukaemia developed into blastic transformation showing a novel NUP98/HOXA11 fusion. British J Haematol 116: 170-172, 2002.

9. Mizoguchi Y, Fujita N, Taki T, Hayashi Y and Hamamoto K: Juvenile myelomonocytic leukemia with $\mathrm{t}(7 ; 11)(\mathrm{p} 15 ; \mathrm{p} 15)$ and NUP98-HOXA11 fusion. Am J Hematol 84: 295-297, 2009. 
10. Martino V, Bianchera A, Reia L, Bussolati O, Fazzina R, Marino F, Montemurro L, Tonelli R, Pession A, Gazzola GC and Sala R: Down-regulation of HOXA4, HOXA7, HOXA10, HOXA11 and MEIS1 during monocyte-macrophage differentiation in THP-1 cells. Mol Med Rep 2: 241-244, 2009.

11. Zhao Y and Potter SS: Functional comparison of the Hoxa 4, Hoxa 10, and Hoxa 11 homeoboxes. Dev Biol 244: 21-36, 2002.

12. Liang DC, Shih LY, Fu JF, Li HY, Wang HI, Hung IJ, Yang CP, Jaing TH, Chen SH and Liu HC: K-Ras mutations and N-Ras mutations in childhood acute leukemias with or without mixed-lineage leukemia gene rearrangements. Cancer 106: 950-956, 2006

13. Grossmann V, Schnittger S, Poetzinger F, Kohlmann A, Stiel A, Eder C, Fasan A, Kern W, Haferlach T and Haferlach C: High incidence of RAS signalling pathway mutations in MLL-rearranged acute myeloid leukemia. Leukemia 27: 1933-1936, 2013.

14. Driessen EM, van Roon EH, Spijkers-Hagelstein JA, Schneider P, de Lorenzo P, Valsecchi MG, Pieters R and Stam RW: Frequencies and prognostic impact of RAS mutations in MLL-rearranged acute lymphoblastic leukemia in infants. Haematologica 98: 937-944, 2013

15. Andersson AK, Ma J, Wang J, Chen X, Gedman AL, Dang J, Nakitandwe J, Holmfeldt L, Parker M, Easton J, et al: The landscape of somatic mutations in infant MLL-rearranged acute lymphoblastic leukemias. Nat Genet 47: 330-337, 2015.

16. Balgobind BV, Zwaan CM, Pieters R and Van den HeuvelEibrink MM: The heterogeneity of pediatric $M L L$-rearranged acute myeloid leukemia. Leukemia 25: 1239-1248, 2011.

17. Barbosa TC, Andrade FG, Lopes BA, de Andrade CF, Mansur MB, Emerenciano M and Pombo-de-Oliveira MS: Impact of mutations in FLT3, PTPN11 and RAS genes on the overall survival of pediatric B cell precursor acute lymphoblastic leukemia in Brazil. Leuk Lymphoma 55: 1501-1509, 2014.

18. Tamai H, Miyake K, Takatori M, Miyake N, Yamaguchi H, Dan K, Shimada T and Inokuchi K: Activated K-Ras protein accelerates human MLL/AF4-induced leukemo-lymphomogenicity in a transgenic mouse model. Leukemia 25: 888-891, 2011.

19. Kim WI, Matise I, Diers MD and Largaespada DA: RAS oncogene suppression induces apoptosis followed by more differentiated and less myelosuppressive disease upon relapse of acute myeloid leukemia. Blood 113: 1086-1096, 2009.

20. Ono R, Kumagai H, Nakajima H, Hishiya A, Taki T, Horikawa K, Takatsu K, Satoh T, Hayashi Y, Kitamura T and Nosaka T: Mixed-lineage-leukemia (MLL) fusion protein collaborates with Ras to induce acute leukemia through aberrant Hox expression and Raf activation. Leukemia 23: 2197-2209, 2009.

21. Fu JF, Yen TH, Chen Y, Huang YJ, Hsu CL, Liang DC and Shih LY: Involvement of Gpr125 in the myeloid sarcoma formation induced by cooperating MLL/AF10(OM-LZ) and oncogenic KRAS in a mouse bone marrow transplantation model. Int J Cancer 133: 1792-1802, 2013.

22. Fu JF, Liang ST, Huang YJ, Liang KH, Yen TH, Liang DC and Shih LY: Cooperation of MLL/AF10(OM-LZ) with PTPN11 activating mutation induced monocytic leukemia with a shorter latency in a mouse bone marrow transplantation model. Int J Cancer 140: 1159-1172, 2017.

23. Rojas JM and Santos E: Ras genes and human cancer: Different implications and different roles. Curr Genomics 3: 2002.

24. Schubbert S, Lieuw K, Rowe SL, Lee CM, Li X, Loh ML, Clapp DW and Shannon KM: Functional analysis of leukemia-associated PTPN11 mutations in primary hematopoietic cells. Blood 106: 311-317, 2005.

25. Yang Z, Li Y, Yin F and Chan RJ: Activating PTPN11 mutants promote hematopoietic progenitor cell-cycle progression and survival. Exp Hematol 36: 1285-1296, 2008.

26. Fu JF, Hsu CL and Shih LY: MLL/AF10(OM-LZ)-immortalized cells expressed cytokines and induced host cell proliferation in a mouse bone marrow transplantation model. Int J Cancer 126: $1621-1629,2010$

27. Valk PJ, Verhaak RG, Beijen MA, Erpelinck CA, Barjesteh van Waalwijk van Doorn-Khosrovani S, Boer JM, Beverloo HB Moorhouse MJ, van der Spek PJ, Löwenberg B and Delwel R: Prognostically useful gene-expression profiles in acute myeloid leukemia. N Engl J Medicine 350: 1617-1628, 2004.
28. Wouters BJ, Löwenberg B, Erpelinck-Verschueren CA, van Putten WL, Valk PJ and Delwel R: Double CEBPA mutations, but not single CEBPA mutations, define a subgroup of acute myeloid leukemia with a distinctive gene expression profile that is uniquely associated with a favorable outcome. Blood 113: 3088-3091, 2009

29. Haferlach T, Kohlmann A, Wieczorek L, Basso G, Kronnie GT, Béné MC, De Vos J, Hernández JM, Hofmann WK, Mills KI, et al: Clinical utility of microarray-based gene expression profiling in the diagnosis and subclassification of leukemia: Report from the international microarray innovations in leukemia study group. J Clin Oncol 28: 2529-2537, 2010

30. Balgobind BV, Hollink IH, Arentsen-Peters ST, Zimmermann M, Harbott J, Beverloo HB, von Bergh AR, Cloos J, Kaspers GJ, de Haas V, et al: Integrative analysis of type-I and type-II aberrations underscores the genetic heterogeneity of pediatric acute myeloid leukemia. Haematologica 96: 1478-1487, 2011.

31. Livak KJ and Schmittgen TD: Analysis of relative gene expression data using real-time quantitative PCR and the 2(-Delta Delta C(T)) method. Methods 25: 402-408, 2001.

32. Leonetti A, Baroli G, Fratini E, Pietropaoli S, Marcoli M, Mariottini P and Cervelli M: Epileptic seizures and oxidative stress in a mouse model over-expressing spermine oxidase. Amino Acids 52: 129-139, 2020.

33. Fu JF, Yen TH, Huang YJ and Shih LY: Ets1 plays a critical role in MLL/EB1-mediated leukemic transformation in a mouse bone marrow transplantation model. Neoplasia 21: 469-481, 2019.

34. Heuser M, Wingen LU, Steinemann D, Cario G, von Neuhoff $N$, Tauscher M, Bullinger L, Krauter J, Heil G, Döhner H, et al: Gene-expression profiles and their association with drug resistance in adult acute myeloid leukemia. Haematologica 90: 1484-1492, 2005

35. Sun Y, Zeng C, Gan S, Li H, Cheng Y, Chen D, Li R and Zhu W: LncRNA HOTTIP-Mediated HOXA11 expression promotes cell growth, migration and inhibits cell apoptosis in breast cancer. Int J Mol Sci 19: 472, 2018.

36. Guo X, Yan B and Qiu Y: HOXA11 regulates chemoresistance by modulating p53 gene expression in acute myeloid leukemia. Blood 134: 5182-5182, 2019.

37. Fiegl H, Windbichler G, Mueller-Holzner E, Goebel G, Lechner M, Jacobs IJ and Widschwendter M: HOXA11 DNA methylation--a novel prognostic biomarker in ovarian cancer. Int J Cancer 123: 725-729, 2008.

38. Hwang JA, Lee BB, Kim Y, Park SE, Heo K, Hong SH, Kim YH, Han J, Shim YM, Lee YS and Kim DH: HOXA11 hypermethylation is associated with progression of non-small cell lung cancer. Oncotarget 4: 2317-2325, 2013.

39. Cui Y, Gao D, Linghu E, Zhan Q, Chen R, Brock MV, Herman JG and Guo M: Epigenetic changes and functional study of HOXA11 in human gastric cancer. Epigenomics 7: 201-213, 2015.

40. Li Q, Chen C, Ren X and Sun W: DNA methylation profiling identifies the HOXA11 gene as an early diagnostic and prognostic molecular marker in human lung adenocarcinoma. Oncotarget 8 : 33100-33109, 2017.

41. Se YB, Kim SH, Kim JY, Kim JE, Dho YS, Kim JW, Kim YH, Woo HG, Kim SH, Kang SH, et al: Underexpression of HOXA11 is associated with treatment resistance and poor prognosis in glioblastoma. Cancer Res Treat 49: 387-398, 2017.

42. Wang L, Cui Y, Sheng J, Yang Y, Kuang G, Fan Y, Jin J and Zhang Q: Epigenetic inactivation of HOXA11, a novel functional tumor suppressor for renal cell carcinoma, is associated with RCC TNM classification. Oncotarget 8: 21861-21870, 2017.

43. Xia B, Shan M, Wang J, Zhong Z, Geng J, He X, Vu T, Zhang D and Pang D: Homeobox A11 hypermethylation indicates unfavorable prognosis in breast cancer. Oncotarget 8: 9794-9805, 2017.

This work is licensed under a Creative Commons Attribution-NonCommercial-NoDerivatives 4.0 International (CC BY-NC-ND 4.0) License. 